\title{
Blazars from the CLASS Survey
}

\author{
M. J. M. Marchã \\ CAAUL, Observatório Astronómico de Lisboa, Tapada da Ajuda, \\ 1349-018 Lisboa, Portugal
}

\author{
A. Caccianiga \\ CAAUL, Observatório Astronómico de Lisboa, Tapada da Ajuda, \\ 1349-018 Lisboa, Portugal
}

\begin{abstract}
.
This paper presents preliminary results from the CLASS blazar sample; the aim is to study the cosmological properties of radio selected BL Lacs and their closest relatives, the other low radio luminosity flat spectrum sources.
\end{abstract}

\section{Introduction}

The term blazar is typically used to group together two types of Active Galactic Nuclei (AGNs): the Flat Spectrum Radio Quasars (FSRQs) and the BL Lac objects. Despite the dichotomy in the type of optical spectra and luminosity range observed, the 2 types of sources show a Spectral Energy Distribution (SED) which is dominated by non-thermal processes. In this work we are primarily interested in the low-power tail of the blazar class, typically made up of BL Lac objects, where many important issues related to the unified models are expected to find a solution. In particular, the beaming model, which unifies BL Lacs and FR I, predicts some important features of the BL Lac luminosity function (LF) that should be observed in the low-power range $\left(\mathrm{P}<10^{24} \mathrm{~W} \mathrm{~Hz}^{-1}\right)$. We have thus created a new radio survey of flat-spectrum sources, the CLASS blazar survey, with the specific aim of exploiting the low-power tail of the blazar class. In this paper we present some preliminary results about the cosmological properties of BL Lacs as derived from this survey.

\section{The Sample}

The CLASS blazar survey consists of 325 flat-spectrum radio sources selected according to the following criteria:

1) $35^{\circ} \leq \delta \leq 75^{\circ}$ and $\left|b^{I I}\right| \geq 20^{\circ}$

2) $S_{5 G H z} \geq 30 \mathrm{mJy}$

3) flat radio spectrum, i.e. $\alpha_{1.4}^{4.8} \leq 0.5\left(\mathrm{~S}_{\nu} \propto \nu^{-\alpha}\right)$

4) red magnitude (corrected) $\leq 17.5$ 
The details of the selection, as well as the radio properties of the sample, are discussed in Marchã et al. (2001), whereas the spectroscopic classification obtained thus far is presented in Caccianiga et al. (2001). In particular, $70 \%$ of the sources have been spectroscopically classified into 3 broad categories:

1) Type 0 - objects with weak or no emission lines (39\%).

2) Type 1 - objects with broad emission lines (46\%).

3) Type 2 - objects with narrow emission lines (15\%).

This paper concentrates on the preliminary results based on the 88 sources of Type 0 identified so far in the CLASS blazar survey.

\section{The Type 0 Classification}

An object is classified as a Type 0 if its optical spectrum shows only emission lines with Equivalent Width $(\mathrm{EW}) \leq 5 \AA$. This class includes both BL Lacs and PEGs (Passive Elliptical Galaxies). The distinction between the 2 depends on the value of the CaII break $(\Delta)$ at $4000 \AA$, which is defined in Dressler \& Shectman (1987).

Since a typical elliptical galaxy shows values of $\Delta$ between $40 \%$ and $60 \%$, a significantly lower value is usually interpreted as an indication of the presence of an extra source of continuum, i.e., a BL Lac nucleus. A limiting value of $\Delta \leq$ $25 \%$ was proposed by Stocke et al. (1991) in order to separate BL Lacs from the remaining type 0 sources. However, it has since been proposed that such a restrictive value may indeed miss weak BL Lacs simply because the ratio of optical emission between the BL Lac and its host galaxy would be too small to be recognized. As a consequence, an "expanded" classification of BL Lac has been used in the selection of new samples of BL Lacs (Marchã et al. 1996, Laurent-Muehleisen et al. 1998, Caccianiga et al. 1999, Perlman et al., 1998), Here we adopt this 'expanded' classification and divide the type 0 sources into 3 categories according to 3 regimes of $\Delta$ :

- "Classical" BL Lacs: $\Delta \leq \mathbf{2 5 \%}$

- BL Lac candidates: $25 \%<\Delta<40 \%$

- PEGs: $\Delta \geq 40 \%$

The imposition of a sharp separation between BL Lacs (and BL Lac candidates) and PEGs is somewhat arbitrary and unlikely to be related to the intrinsic properties of the active nucleus. In fact, the measured contrast of a source is inversely proportional to the strength of the non-thermal contribution from the AGN, which means that as the AGN gets weaker, the measured contrast will get larger. Hence, the deeper the survey, the more important is the choice of contrast used in the selection of BL Lacs. A value for this quantity that is too limiting can introduce significant incompleteness in the final sample, as is widely discussed in Browne \& Marchã (1993) and Marchã \& Browne (1995). More quantitatively, by using the formalism discussed in these papers, we predict that a high fraction (43\%) of the low-power (down to $10^{23} \mathrm{~W} \mathrm{~Hz}^{-1}$ ) BL Lacs are not expected to produce a significant reduction of $\Delta$ (i.e. leaving $\Delta \geq 40 \%$ ). This value is in good agreement with the observed fraction of PEGs found in the survey $(45 \%)$.

It is clear that with the currently available data it is not possible to prove or disprove that all (or a fraction) of the PEGs found in the CLASS blazar 
sample actually contain a hidden BL Lac nucleus in their cores. However, the alternative to this scenario is to invoke a new population of low luminosity, coredominated, flat radio spectrum sources which so far has no place in the current unified scheme for radio-loud objects. We are thus faced with two scenarios: one where PEGs hide weak BL Lac nuclei, and another where they are an entirely different population. The consequences for the statistical properties of BL Lacs will be different in both scenarios, and for that reason, the next Section dealing with the cosmological properties will treat both situations separately.

\section{Cosmological Properties}

The cosmological evolution of BL Lacs has been a matter of debate over the last decade, and it is still considered an open problem. At the origin of the debate are the opposing results obtained about ten years ago when using the only 2 statistically complete samples of BL Lacs available at the time, one selected at radio frequencies (1 Jy sample; Stickel et al. 1991) and the other in the X-ray (the EMSS-C sample; Morris et al. 1991). In the case of the radioselected sample, the result was consistent with a population that suffered no evolution, or mildly positive, whereas in the case of the X-ray selected sample, the result was clearly indicative of a population that evolves negatively. More recent results (Bade et al. 1998; Giommi, Menna \& Padovani 1999) based on ROSAT data seem to confirm this discrepancy showing negative evolution for High-energy peaked BL Lacs (HBL), and no evolution for the Low-energy peaked BL Lacs (LBL). On the other hand, the samples selected by combining radio and X-ray data (e.g. the REX survey, Caccianiga et al. 1999 and the DXRBS survey, Perlman et al. 1998) are not finding strong evidence for either negative or positive evolution (Padovani 2001, Caccianiga et al. 2002 and Caccianiga et al, this conference). Even if some attempts at explaining these results in terms of different selection effects have been done (Giommi et al. 2001), the general picture is still very unclear.

The CLASS blazar survey offers an independent tool to asses the evolutionary properties of BL Lacs although, to date, the missing $30 \%$ of identifications prevent us from drawing firm conclusions. Nevertheless, assuming that the missing $30 \%$ are distributed like the remaining objects, we have applied the $V_{e} / V_{a}$ test (Avni \& Bahcall 1980) to estimate the cosmological evolution of the CLASS BL Lacs. The results can be found in Table 1.

$$
\begin{array}{cccc} 
& \text { BL Lacs (48) } & \text { PEGs (40) } & \text { All type 0 (88) } \\
\left\langle V_{e} / V_{a}\right\rangle & 0.61 & 0.48 & 0.55 \\
& \pm 0.04 & \pm 0.05 & \pm 0.03
\end{array}
$$

Table 1. Mean $V_{e} / V_{a}$ values for the entire sample of type 0 sources; the numbers in brackets correspond to the number of sources in each category

The value of $\left\langle V_{e} / V_{a}\right\rangle=0.61$ for BL Lacs (including the BL Lac candidates) was obtained by assigning a redshift of $z=0.26$ for the 25 featureless objects. This redshift corresponds to the mean of the remaining $23 \mathrm{BL}$ Lacs (with redshift) in the sample. It is important to note, however, that the value 

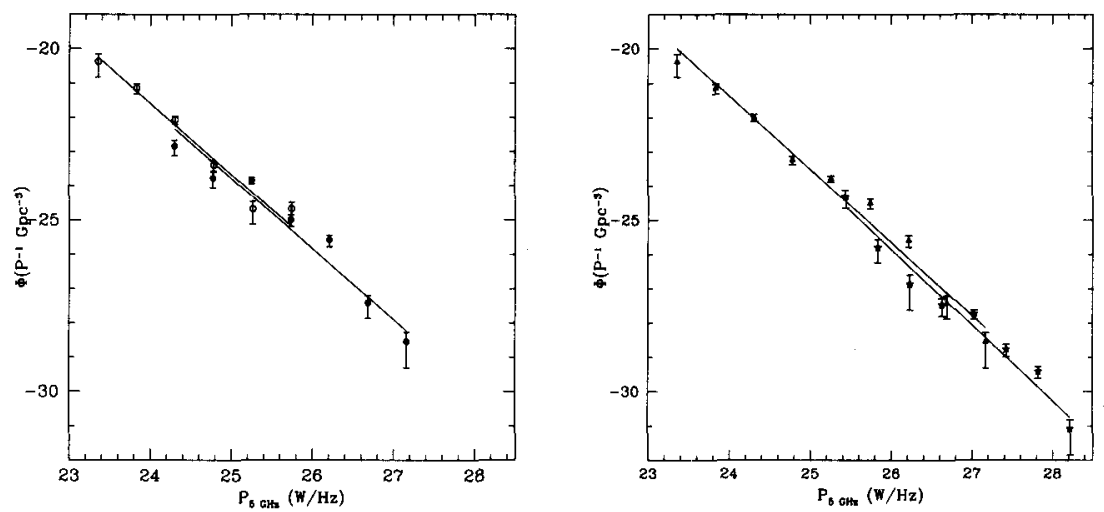

Figure 1. Left: The RLF of BL Lacs (filled circles) and PEGs (open circles). Right: The RLF of all the type 0 (triangles) compared to the BL Lac RLF from the $1 \mathrm{Jy}$ sample (stars)

of $\left\langle V_{e} / V_{a}\right\rangle$ does not change very much by changing this assumption. For instance, using a much lower or higher mean redshift of $z=0.1$ or $z=0.5$ the $\left\langle V_{e} / V_{a}\right\rangle$ varies from 0.59 to 0.62 , respectively. For the remainder of the analysis, the BL Lacs without a measured redshift are assigned $z=0.26$.

The values of $\left\langle V_{e} / V_{a}\right\rangle$ presented in Table 1 show that PEGs are consistent with a scenario of no evolution, while the $\left\langle V_{e} / V_{a}\right\rangle$ of BL Lacs (without PEGs) is significantly $(2.8 \sigma)$ away from the case of no evolution. This result has interesting consequences for the discussion of the evolution of BL Lacs. If BL Lacs (including BL Lac candidates) alone are considered, then the above results give statistical evidence for mildly positive cosmological evolution for this class of objects. However, if PEGs are the population of 'diluted' BL Lacs, as discussed in the previous section, then $V / V_{\max }$ becomes consistent with a non-evolving population.

\subsection{The Radio Luminosity Function (RLF)}

The study of the RLF of radio-loud objects is particularly interesting because, according to the unified schemes, there should be a relationship between the LF of beamed and unbeamed (parent) populations. The effect of beaming on the LF was studied in detail by Urry \& co-workers (see Urry \& Padovani, 1995 and references therein for a review). In summary, if the unbeamed LF is well represented by a power-law with a given slope within a luminosity range, then the effect of beaming is to produce a break in the LF at a luminosity $L_{b r e a k}$ that depends on the Doppler factor. The slope of the beamed LF will be the same as that of the parent population above this $L_{b r e a k}$ but it will flatten below this luminosity.

The left panel of Fig. 1 shows the RLF for the BL Lacs (including BL Lac candidates as filled circles), and PEGs (open circles) of the CLASS blazar sample. (The LF discussed here assumes a no-evolution scenario, a $70 \%$ normalization of the surveyed area in order to account for the $70 \%$ spectroscopic 
identification obtained so far, and a bin size $\Delta \log L=0.5$.) The $2 \mathrm{LFs}$ cover $\sim$ 4 orders of magnitude in radio luminosity, with the PEGs extending to as low as $\sim 10^{23} \mathrm{~W} \mathrm{~Hz}^{-1}$. There is good agreement between both LFs over their overlapping region. A least squares fit to each data set gives a slope of $-2.00( \pm 0.19)$ for the PEGs, and $-1.96( \pm 0.20)$ for BL Lacs. Since the beaming model of Urry \& Padovani (1995), based on the data of the $1 \mathrm{Jy}$ BL Lac data, predicts a flattening of the beamed LF at about $L \sim \times 10^{25} \mathrm{~W} \mathrm{~Hz}^{-1}$, we also investigate this possibility using our data. A possible flattening below $L_{\text {break }} \sim 2 \times 10^{25}$ W $\mathrm{Hz}^{-1}$ is consistent with our BL Lac data. In this case, a least squares fit gives a slope of $-1.06( \pm 0.52)$ below and $-2.47( \pm 0.22)$ above $L_{\text {break }}$. However, even if a flattening of the BL Lac LF is consistent with our data, it is certainly not required.

The right panel of Figure 1 shows the LF for the entire type 0 population (BL Lacs + PEGs) represented by triangles, against that of the 1 Jy sample of BL Lacs which is represented by stars. The concordance between the 2 LFs is remarkable. A least squares fit applied to each set of data gives a slope of $-2.09( \pm 0.11)$ for the type 0 and $-2.18( \pm 0.18)$ for the $1 \mathrm{Jy}$ data.

The 2 LFs overlap over 2 orders of magnitude in luminosity and as mentioned before, the LF for CLASS type 0 extends to 2 orders of magnitude below the luminosities of the $1 \mathrm{Jy}$ BL Lacs. Nevertheless, the slopes found for both LFs are very similar, and in this case there is little evidence for a flattening of the RLF below $\sim 10^{25} \mathrm{~W} / \mathrm{Hz}$. Hence, if weak BL Lac nuclei do exist in the core of PEGs, then the flattening of the RLF is either very mild, or it occurs at lower luminosities. In either case, there are consequences for the beaming parameters to be considered in the BL Lac population.

\section{Conclusion}

In this paper the preliminary results concerning the cosmological properties of the 88 type 0 objects discovered so far in the CLASS blazar survey were briefly discussed. This group of sources contains both BL Lacs and PEGs, and it consists of all the objects without strong emission lines found in the $70 \%$ complete spectroscopic identification process. The analysis considered 2 possible scenarios: one considering that PEGs hide BL Lac nuclei, and another where they do not. When BL Lacs were considered alone, then mild positive evolution was found. However, this trend disappeared under the hypothesis that PEGs harbor BL Lacs. The RLF was also investigated under the 2 possible scenarios, and when kept separately, the data yielded RLFs of similar slopes for BL Lacs and PEGs. Even though there was no need to invoke a break in the LF for BL Lacs, a flattening at low radio luminosities could not be excluded. The position of this eventual flattening and the respective slopes below and above the $L_{b r e a k}$ are consistent with those found by Urry \& Padovani (1995). Nevertheless, if BL Lacs and PEGs are considered together, the resulting RLF extends to 2 orders of magnitude below the luminosities sampled by the $1 \mathrm{Jy}$ sample of BL Lacs, and the 2 LFs are well represented by simple power-laws of similar slopes. In this scenario no flattening is observed in the LF for the type 0 objects of the CLASS sample. 
The fact that the spectroscopic identification level is only $70 \%$ complete means that the discussion presented here is preliminary and that further data are needed. Apart from the completion of the identification process, further optical, radio and $\mathrm{X}$-ray data are being considered in order to establish on a firmer basis whether PEGs do indeed harbor BL Lacs, or whether they constitute a new population of flat spectrum radio sources. The answer to this question will undoubtedly have repercussions on the statistical properties of the BL Lac class.

\section{References}

Avni, Y. \& Bahcall, J.N. 1980, ApJ, 235, 694

Bade, N., Beckmann, V., Douglas, N. G., Barthel, P. D., Engels, D., Cordis, L., Nass, P., Voges, W. 1998, å, 334, 459

Browne, I.W.A. \& Marchã, M.J.M. 1993, MNRAS, 261, 795

Caccianiga, A., Maccacaro, T., Wolter, A., della Ceca, R., Gioia, I. 1999, ApJ, 513,51

Caccianiga, A., Marchã, M. J. M., Antón, S., K.-H, M., Neeser, M. J. 2001, MNRAS, in press

Caccianiga, A., Maccacaro, T., Wolter, A., della Ceca, R., Gioia, I. 2002, ApJ, in press

Giommi, P., Menna, M. T., Padovani, P. 1999, MNRAS, 310, 465

Giommi, P., Pellizoni, A., Perri, M., Padovani, P. 2001, in ASP Conf. Ser. Vol. 227, Blazar Demographics and Physics, ed. P. Padovani \& C. M. Urry, (San Francisco; ASP), 227

Laurent-Muehleisen, S. A., Kollgaard, R. I., Ciardullo, R., Feigelson, E. D., Brinkmann, W., Siebert, J. 1998, ApJS, 118, 127

Marchã, M. J. M. \& Browne, I. W. A. 1995, MNRAS, 275, 954

Marchã, M. J. M., Browne, I. W. A., Impey, C. D., Smith, P. S. 1996, MNRAS, 281,425

Marchã, M. J., Caccianiga, A., Browne, I. W. A., Jackson, N. 2001, MNRAS, 326,1455

Morris, S. L., Stocke, J. T., Gioia, I. M., Schild, R. E., Wolter, A., Maccacaro, M., della Ceca, R. D. 1991, ApJ, 380, 49

Padovani, P., 2001, in ASP Conf. Ser. Vol. 227, Blazar Demographics and Physics, ed. P. Padovani \& C. M. Urry, (San Francisco; ASP), 163

Perlman, E., Padovani, P., Giommi, P., Sambruna, R., Jones, L. R., Tzioumis, A., Reynolds, J. 1998, AJ, 115, 1253

Stickel, M., Padovani, P., Urry, C. M., Fried, J. W., Kühr, H. 1991, ApJ, 374, 431

Stocke, J. T., Morris, S. L., Gioia, I., Maccacaro, T., Schild, R. E., Wolter, A., Fleming, T. A., Henry, J. P. 1991, ApJS, 76, 813

Urry, C.M. \& Padovani, P., 1995 PASP, 107, 803 